\title{
Direct reprogramming of adult cells: avoiding the pluripotent state
}

This article was published in the following Dove Press journal:

Stem Cells and Cloning:Advances and Applications

15 February 2014

Number of times this article has been viewed

\author{
Sophia Kelaini \\ Amy Cochrane \\ Andriana Margariti \\ Centre for Experimental Medicine, \\ School of Medicine, Dentistry \\ and Biomedical Sciences, Queen's \\ University Belfast, Institute of Clinical \\ Sciences, Belfast, UK
}

\begin{abstract}
The procedure of using mature, fully differentiated cells and inducing them toward other cell types while bypassing an intermediate pluripotent state is termed direct reprogramming. Avoiding the pluripotent stage during cellular conversions can be achieved either through ectopic expression of lineage-specific factors (transdifferentiation) or a direct reprogramming process that involves partial reprogramming toward the pluripotent stage. Latest advances in the field seek to alleviate concerns that include teratoma formation or retroviral usage when it comes to delivering reprogramming factors to cells. They also seek to improve efficacy and efficiency of cellular conversion, both in vitro and in vivo. The final products of this reprogramming approach could be then directly implemented in regenerative and personalized medicine.
\end{abstract}

Keywords: ESCs, iPS, PiPS, reprogramming, transdifferentiation, miRNA

\section{Changing the cell fate}

The traditional view of cell differentiation initially involved the classic concept of an uncommitted cell differentiating into a committed tissue-specific type. ${ }^{1}$ Since then, however, this view has been reassessed as it has been proven that it is entirely possible to change a differentiated cell's fate toward pluripotency or toward an entirely different cell type. ${ }^{1}$ Somatic cells can be reprogrammed into pluripotent cells using a variety of methods ${ }^{2}$ that include somatic cell nuclear transfer, cell fusion, culture-induced reprogramming using cell extracts, and direct reprogramming, the latter being the focus of this review. More particularly, direct reprogramming of a cell is a complex process that involves a wide variety of methods and reprogramming factors (Table 1), either alone or in combination, and whose mechanisms of action still remain unclear. Direct reprogramming of cells into a different state (either pluripotent or somatic) offers one of the most promising developments in the field of regenerative medicine, and the possibilities to realize this immense potential in clinical and therapeutic applications need to be examined in more depth.

\section{Regenerative medicine, stem cells, and tissue regeneration}

Pluripotent stem cells are able to differentiate into nearly all types of cells within the body. ${ }^{3}$ They are undifferentiated cells that can self-renew and proliferate to undifferentiated cells both in vitro and in vivo as well as into mature specialized cells. ${ }^{4}$ This offers the significant prospect for cell-based therapies to repair tissues or organs destroyed by injury, degenerative disease, aging, or cancer. ${ }^{5-7}$ A number of different types of pluripotent 
Table I Examples of different types of cellular reprogramming and their corresponding reprogramming factors used for directing cell fate switch

\begin{tabular}{|c|c|c|}
\hline Cell origin & $\begin{array}{l}\text { Derived } \\
\text { cell type }\end{array}$ & References \\
\hline \multicolumn{3}{|l|}{ Examples of reprogramming } \\
\hline Fibroblasts & iPS cells & \\
\hline Oct4, Sox2, KIf4, c-MYC & & Takahashi'3 \\
\hline Oct4, Sox2 + valproic acid & & Huangfu, ${ }^{80}$ \\
\hline Oct4, Sox2, c-MYC + & & Lyssiotis $^{83}$ \\
\hline kenpaullone & & $\mathrm{Li}^{82}$ \\
\hline \multicolumn{3}{|l|}{ Oct4, Klf4 + CHIR9902I } \\
\hline (MEFs) & & \\
\hline \multicolumn{3}{|c|}{ Examples of transdifferentiation and direct reprogramming } \\
\hline Fibroblasts & Muscle & \\
\hline MyoD & & Davis $^{34}$ \\
\hline Pancreatic (exocrine) & Pancreatic & \\
\hline Ngn3, PdxI, Mafa & (beta cells) & Zhou ${ }^{44}$ \\
\hline Fibroblasts & Neurons & \\
\hline Ascll, Brn2, Mytll & & Vierbuchen ${ }^{26}$ \\
\hline Fibroblasts & Cardiomyocytes & \\
\hline Gata4, Mef2c, Tbx5 & & $\operatorname{leda}^{27}$ \\
\hline Gata4, Mef2c, Tbx5, Hand2 & & Song ${ }^{45}$ \\
\hline Gata4, Mef2c, Tbx5, VEGF & & Mathison ${ }^{49}$ \\
\hline Mef2c, Myocardin, and Tbx5 & & Protze $^{46}$ \\
\hline Fibroblasts & Cardiomyocytes & \\
\hline $\begin{array}{l}\text { Myocardin, miR-I, } \\
\text { miR-I33, GHMT }\end{array}$ & & $\mathrm{Nam}^{29}$ \\
\hline Fibroblasts & Endothelial cells & \\
\hline $\begin{array}{l}\text { Oct4, Sox2, KIf4, c-MYC } \\
\text { (4-day partial reprogramming) }\end{array}$ & & Margariti $^{55}$ \\
\hline $\begin{array}{l}\text { Oct4, Sox2, KIf4, c-MYC } \\
\text { (Short reprogramming) }\end{array}$ & & $\mathrm{Li}^{62}$ \\
\hline Amniotic & Endothelial cells & \\
\hline ETV2, FLII, ERGI & & Ginsberg ${ }^{64}$ \\
\hline
\end{tabular}

Abbreviations: Ascll, achaete-scute homolog I; Brn2, (brain-2) also called Pouf3 or POU class 3 homeobox 2 ;c-MYC, c-myelocytomatosis oncogene; ERG I, epilepsyrelated gene I; ETV2, ETS translocation variant 2; FLII, Fli-I proto-oncogene; ETS, transcription factor (previous name: Friend leukemia virus integration I); GHMT, Hand2 + GMT; Hand2, heart- and neural crest derivatives-expressed protein 2; iPS, induced pluripotent stem; KIf4, Kruppel-like factor 4; Mafa, v-maf avian musculoaponeurotic fibrosarcoma oncogene homolog A; Mef2c, myocyte-specific enhancer factor 2C; MEFs, mouse embryonic fibroblasts; miR-I, microRNA-I; miR133, microRNA-133; MyoD, myogenic differentiation; Myt II, myelin transcription factor I-like; Ngn3, neurogenin 3; Oct4, octamer-binding protein; PdxI, pancreatic and duodenal homeobox I; Sox2, sex determining region Y-box containing gene 2; Tbx5, T-box transcription factor; VEGF, vascular endothelial growth factor.

stem cells have been described according to their characteristics. These include embryonic stem cells (ESCs), which are able to differentiate into derivatives of the three germ layers: endoderm, mesoderm, and ectoderm. ${ }^{8}$ Other types of stem cells include somatic stem cells found in differentiated tissues, ${ }^{9}$ fetal stem cells derived from the fetus, ${ }^{10}$ and mesenchymal stem cells, a type of multipotent stromal cell. ${ }^{11}$ It is important to note that one of the most commonly-used assays for demonstrating pluripotency is forming teratomas. ${ }^{12}$

The recent discovery of induced pluripotent stem (iPS) cells also offers a promising strategy to generate therapeutically-relevant numbers of patient-specific cells. ${ }^{13}$ iPS cells are pluripotent stem cells that have been artificially derived from nonpluripotent differentiated somatic cells and share many common features with ESCs. However, some differences were also shown to exist between them. Namely, Chin et $\mathrm{al}^{14}$ showed that a small set of genes is differentially and continuously expressed between several iPS and ESC lines. In contrast to this finding however, other groups reported that differences between the expression profiles between these cell types are not consistent and may be a result of different cell culture conditions. ${ }^{15}$ Despite these contrasting reports however, the common transcriptome pattern that arises by looking at these differences seems to involve iPS cells not effectively silencing somatic cell gene expression and/or not inducing ESC-specific genes to the same level as ESCs. ${ }^{16}$

The most common source of iPS cells is usually fibroblasts but other sources have also been reported such as hepatocytes and mature B cells. ${ }^{17}$ More specifically, fibroblasts can be reprogrammed to stable self-renewing iPS cells which resemble ESCs by enforced expression of a cocktail of transcription factors consisting of octamer-binding protein (Oct4), SRY-box containing gene 2 (Sox2), Kruppel-like factor 4 (Klf4), and c-myelocytomatosis oncogene (c-Myc). iPS cells can be generated, expanded, and then differentiated into various cell types such as endothelial cells (ECs), neurons, and cardiomyocytes for in vitro studies or, ultimately, cell therapy. ${ }^{18-20}$ Moreover, constructs for molecular imaging can also be engaged to facilitate tracking of, for example, transplanted ECs in vivo. ${ }^{21}$ In addition, patient-specific cells can be generated to study the effects of genetic or epigenetic changes in patients. ${ }^{22}$ Therefore, there is huge potential to employ these cells for personalized medicine and vascular therapy, while overcoming the major disadvantages of using adult cells from blood vessels, which may include limited proliferation capacity and susceptibility to cellular senescence..$^{23}$

\section{Limitations in ESC/iPS cell technology}

Although, the use of iPS cells to derive a desired cell type may overcome these difficulties, a limitation of induced pluripotency is the length of time it takes to first reprogram the cells and then subsequently direct them to the preferred fate. Since the protocols to generate iPS cells include a number of stages, the efficiency with which the final cell type is generated can be low. In addition, a number of concerns about the fidelity and safety of iPS/ESC-derived cells need to be addressed before these cells could be used clinically. ${ }^{24}$ More particularly, several criteria must be met 
when it comes to iPS cells to confirm their developmental potential; these criteria include successful differentiation in vitro and the expression of specific cell surface markers as well as teratoma formation. ${ }^{2}$ The tumor formation should be able to induce differentiation to all three germ layers. In addition, chimera formation after iPS injection into mouse diploid blastocysts is another necessary test. In this manner, iPS cells should greatly contribute toward tissue and tetraploid embryo development. ${ }^{2}$

Moreover, the lack of availability of unfertilized oocytes from human volunteers poses a technical challenge in isolation and expansion of human ESCs. ${ }^{2}$ Regardless of low availability and the dependency on volunteer donation, the technique's efficiency is generally low. ${ }^{25}$ Taking into account all these limitations related to the use and generation of iPS cells, other ways of reprogramming cells have been considered that involve a more direct conversion between cellular types while avoiding the pluripotent stage and its related disadvantages.

\section{Transdifferentiation}

An alternative way to bypass the pluripotent ESCs/iPS stage is through transdifferentiation. Studies revealed that fibroblasts could be converted to several lineages including neurons,${ }^{26}$ cardiomyocytes, ${ }^{27}$ and hepatocytes ${ }^{28}$ by ectopic expression of multiple lineage-specific transcription factors or microRNAs (miRNAs). ${ }^{29}$ Importantly, this approach had also been applied in vivo using a number of lineage-specific transcription factors. ${ }^{30}$

\section{History of cellular reprogramming and transdifferentiation}

The differentiated state has been the subject of multiple experimental procedures that goes as far back as the 1950s. Previous studies have revealed that differentiation into a specific cell type can be achieved simply by activating one or a few relevant genes. These key genes (master genes) are usually the first genes activated in specialization pathways, and they are also needed in eliciting responses from even more genes during development. Indeed, in Drosophila experiments, ${ }^{31,32}$ master gene overexpression activated genes found in other cell types, affecting cells and their eventual fate..$^{32,33}$ Master genes are also found in mammals. ${ }^{34}$ Traditional approaches in reprogramming have included nuclear transfer, fusion, and transcriptional factor-based transduction. All of these approaches eventually revealed that the state of differentiation is not unchanging; on the contrary, it is flexible, reversible, and adjustable. ${ }^{13,33,35,36}$ Initial, ground breaking cloning experiments in frogs during the 1950s revealed that the nuclear transfer from blastocysts to enucleated oocytes gave rise to cloned organisms and delivered one of the first definite indications of nonpermanent gene silencing. ${ }^{37} \mathrm{~A}$ few decades later, in the 1980s, the first transcription factor-based reprogramming experiments took place. Transfection of Myogenic differentiation factor (MyoD), normally expressed in skeletal muscle, was reported to convert embryonic mouse fibroblasts into muscle cells by forcing the cells to express this specific gene. ${ }^{34} \mathrm{After}$ this initial success, MyoD was later used in many more reprogramming experiments, successfully converting immature chondrocytes, smooth muscle cells, and retinal cells into muscle cells. ${ }^{38}$ However, it could not successfully convert other types of cells such as hepatocytes into muscle. ${ }^{39}$

Another important reprogramming factor was discovered in the 1990s and it was specifically related to hematopoiesis: globin transcription factor 1 (Gata-1) could induce avian monocyte precursors into erythrocytes, eosinophils, and megakaryocytes. ${ }^{40}$ In 2004 it was also shown that descendants derived from common lymphoid progenitors could also be converted to descendants of common myeloid progenitors. More specifically, it was shown that B cells could be reprogrammed into macrophages through expression of CCAAT-enhancer-binding protein (C/EBP) transcription factors. The gene expression pattern and functional properties of the reprogrammed cells were tested by infecting CD19+ mouse bone marrow cells with $\mathrm{C} / \mathrm{EBPa} / \mathrm{humanCD} 4$ virus and its corresponding control. Cells were sorted and the subsequent molecular and morphological analysis revealed the functionality of the in vitro reprogrammed macrophages. ${ }^{41}$ C/EBP inhibits paired box protein-5 (Pax5), a transcription factor which can strengthen a B cell's commitment. ${ }^{41,42}$ Once the Pax 5 gene is deleted, $\mathrm{B}$ cells dedifferentiate and turn into common lymphoid progenitor-like cells, which can in turn be differentiated into T cells. ${ }^{43}$ However, this is not considered direct reprogramming per se, as it requires passing through the lymphoid progenitor cell state first.

More recently, in 2008, Zhou et al ${ }^{44}$ demonstrated that by overexpressing neurogenin 3 , pancreatic and duodenal homeobox 1(Pdx1), and v-maf avian musculoaponeurotic fibrosarcoma oncogene homolog A (Mafa) (NPM) exocrine pancreas cells were converted into insulin-secreting beta celllike cells. This was achieved after screening more than 1,000 transcription factors, resulting in the identification of a number of genes uniquely expressed in mature beta-cells. Out of these, nine proved to be of great importance in the development of the pancreas. Ultimately, it was found that the combination of transcription factors that worked in the best possible way was NPM. 
Functionality was tested by injecting an adult mouse pancreas with a combination of NPM viruses (pAd-M3); 1 month later numerous insulin-positive cells appeared outside of islets. ${ }^{44}$

Using a similar strategy, another study ${ }^{27}$ achieved a breakthrough in reprogramming mouse fibroblast cells into beating cardiomyocyte-like cells; it was reported that fibroblasts could be directly converted into cardiomyocyte-like cells through overexpression of cardiac-specific factors both in vivo and in vitro (GATA binding protein 4 [Gata-4], myocyte-specific enhancer factor $2 \mathrm{C}$ [Mef2c], and T-box 5 [Tbx5] [GMT]). ${ }^{27}$ Reprogramming of fibroblasts into functional neuron cells was also reported after forced expression of achaete-scute homolog 1 (Ascl1), transcription factor brain-2 (also called Pouf3 or POU class 3 homeobox 2)[Brn2], and myelin transcription factor 1-like (Myt11). ${ }^{26}$ Using lentiviral vectors to infect the fibroblasts, the researchers initially screened for the potential of 19 candidate factors to induce a neuron-like phenotype, and eventually the combination of Ascl1, Brn2, and myelin transcription factor 1-like was determined to be fundamental in neuron conversion. More specifically, even though Ascl1 on its own could still induce some immature neuron-like features, the other two transcription factors were also essential for the conversion to mature functional neuron-like cells expressing proteins that are neuron-specific.

Since the original report on the GMT combination by Ieda et al, ${ }^{27}$ transdifferentiation of fibroblasts to cardiomyocyte-like cells in vitro has been reported by many more groups. Song et al screened for transcription factor combinations and showed that addition of the transcription factor heart- and neural crest derivatives-expressed protein 2 (Hand2) to the GMT factor mix led to in vitro reprogramming of adult mouse tail-tip and cardiac fibroblasts into beating cardiac-like myocytes. ${ }^{45}$ Protze et $\mathrm{a}^{46}$ used an alternative screening approach to evaluate triplet combinations of ten candidate factors using the quantitative polymerase chain reaction and reported induction of a variety of cardiac-specific genes. More specifically, the combination of Mef2c, myocardin, and Tbx5 was shown to upregulate a wider variety of cardiac genes compared to GMT.46,47 Cardiac reprogramming has also been reported to occur in vivo: Song et $\mathrm{al}^{45}$, Jayawardena et $\mathrm{al}^{54}$, and Qian et $\mathrm{al}^{30}$ all used the fibroblast-specific protein 1 promoter; ${ }^{47}$ however, Qian et al additionally used periostin, a fibroblast-specific promoter, as a labeling system. ${ }^{30}$ On the other hand, Song et al used transcription factor 21 or epicardin promoter, which is not found in cardiac myocytes. ${ }^{45,47}$ In support of the earlier studies on GMT, Inagawa et al demonstrated GMT-mediated in vivo reprogramming in infarcted mouse hearts. ${ }^{48}$ In addition, Mathison et al reported that vascular endothelial growth factor could improve
GMT reprogramming efficiency in rat hearts. ${ }^{49}$ In both Qian et al and Song et al's experiments, infarcted hearts showcased significant functional recovery. Even after all of these experiments, however, it is still uncertain which combination of factors demonstrates the biggest efficiency in transdifferentiating to cardiomyocytes, as any comparisons take place by using separate experimental systems and different lab conditions. ${ }^{47}$ In addition, subsequent studies in cardiac reprogramming raised questions. For instance, despite showing that GMT-mediated reprogramming of heart fibroblasts could produce a significant number of $\alpha$-myosin heavy chain-green fluorescent protein ( $\alpha$-MHC-GFP)-positive cells in a short period of time, ${ }^{27}$ very few of the cells within that population expressed cardiac troponin $\mathrm{T}$ or continued spontaneous beating after a few weeks in culture. ${ }^{47}$ On the contrary, spontaneous cardiomyocyte beating was seen at higher occurrence upon differentiation of ESCs and iPS cells. ${ }^{50,51}$ Since then, more recent reports failed to observe spontaneous beating when using lentiviruses to express GMT in cardiac fibroblasts. ${ }^{52}$ This raises the question of whether the original study's cardiomyocytes could have originated from either immature cardiomyocytes or cardiac progenitor cells in the initial heart fibroblast pool. ${ }^{46}$ This is in accordance with some reports of small contaminating populations of cardiomyocytes or progenitor cells, even after sorting purification procedures. ${ }^{53}$ However, despite these limitations, it seems that GMT and other groups of cardiac-specific groups of factors can still push cells toward a cardiomyocyte-like cell fate. ${ }^{46,47,54}$

\section{Limitations}

Cells generated as illustrated above may demonstrate restricted proliferative capacity, limited cell type diversity, and even senescence, ${ }^{55}$ which may in turn substantially compromise their potential application in regenerative therapy. Thus, in striving toward the prospect of generating patientspecific tissues and organs, another approach for regenerative medicine was developed: direct reprogramming resulting in a rejuvenated ${ }^{55}$ cell phenotype, which, just like transdifferentiation, also avoids reaching the pluripotent stage with its related disadvantages.

\section{Direct reprogramming: an emerging alternative strategy}

The procedure of using mature, fully differentiated somatic cells and inducing them toward other cell types while bypassing an intermediate pluripotent state is termed direct reprogramming. Recent advances in this field have shown it to be one of the most promising cellular and tissue generating approaches in regenerative medicine. Today, one of the most 
desired targets in cellular reprogramming is the conversion of committed adult cells into pluripotent ones and also the generation of the preferred adult cell through direct conversion between cellular types.

Before proceeding to discuss "direct reprogramming" it should be noted that sometimes confusion occurs when distinguishing this specific type of reprogramming from conventional direct reprogramming known as "transdifferentiation". For example, both approaches share some common features, such as direct conversion from one cellular type to another and being patient-specific. In addition, both approaches avoid reaching a final pluripotent stage but, on the contrary, they rely on specific signals to help them reach the desired cellular destination. Also for both, such a conversion usually occurs in a short period of time when compared to iPS cell generation. However, the cellular conversion in transdifferentiation is usually the result of overexpression of lineage-specific factors, which pushes the conversion toward the cell lineage of interest. On the contrary, "direct reprogramming" involves the short-term cellular opening of pluripotent-related pathways, which initially drive the cell toward the pluripotent stage, before directing it toward a lineage-specific path.

A complex regulatory network of transcription factors supports expression of cell type-specific genes and suppresses regulators of other lineages to establish and maintain cell fate during development. This stability is likely the result of multilayer combinations of regulation, including epigenetic/ posttranslational modifications, DNA-binding transcription factors, transcriptional coactivators, noncoding RNAs, and chromatin remodeling. ${ }^{56,57}$ As already mentioned, although generally stable in vivo, cell fate can, under certain experimental conditions, be dominantly reprogrammed by forcing expression of transcription factors involved in the establishment and maintenance of a distinct cellular lineage. ${ }^{58}$ Recently however, a new paradigm of direct reprogramming strategy has been devised, which involves "the conversion of one somatic cell type to another through direct reprogramming". 55 We have tested whether at earlier time points during reprogramming it is possible to direct the epigenetically activated cells, which are induced by the reprogramming factors, into lineage-specific cell types such as ECs under defined conditions by skipping pluripotency. ${ }^{55}$

The main principle of this direct reprogramming approach is that conventional iPS cell reprogramming proceeds as a slow, step-wise process. In addition, generating iPS cells requires an extended period of enforced transcription factor expression (8-12 days) and a specific signaling environment.
To this end, fibroblasts were reprogrammed with transient overexpression of the reprogramming factors for 4 days. These cells were named partial-iPS (PiPS) cells (Figure 1). Importantly, PiPS cells responded to different signaling environments (eg, growth factors/cytokines) and were able to direct reprogrammed cell fate decisions. Compared with conventional transdifferentiation, this new method has several advantages, such as the use of a universal transcription factor system and the ability to generate a rejuvenated ${ }^{55}$ mul- $^{-}$ tipotent progenitor cell population, able to differentiate into specific cell types in response to a specific stimulus. Using this method, vascular ECs were generated (Figure 1), which could prove useful in regenerative medicine, ${ }^{59}$ for example, vascular tissue engineering. ${ }^{60}$ The generation of tissue-engineered vascular grafts represents a major breakthrough with enormous potential and clinical applications. ${ }^{61}$ Importantly, when performing functionality tests, these cells did not form tumors when injected into immunodeficient mice since they had not reached a pluripotent stage. More specifically, PiPS cell-derived ECs were proven to be functional in angiogenesis (using an ischemic limb model) and in reendothelialization (using tissue-engineered vessels ex vivo). ${ }^{55}$ In particular, PiPSderived ECs were able to form vascular-like tubes in both in vitro and in vivo experiments in severe combined immunodeficiency mice. ${ }^{55}$ The ability to convert fibroblasts to ECs through direct reprogramming has recently been confirmed. ${ }^{62}$ In addition, PiPS cells are able to differentiate not only toward ECs but also toward smooth muscle cell lineages. ${ }^{63}$ Figure 1 shows the different routes for cellular conversion using reprogramming genes: a) reprogramming to iPS cells using four reprogramming factors followed by differentiation to a specific cell lineage (such as ECs), b) direct reprogramming to PiPS followed by differentiation to a specific cell type, and c) direct conversion (without differentiation) to the desired cell type. It must be noted that in the case of direct conversion, the mechanisms are not yet fully elucidated but may involve specific cell lineage genes and be characterized by low efficiency, senescence, and unknown conversion time (possibly extensive), while in reprogramming methods with a differentiation step, the efficiency is relatively high.

In a similar manner, a previous study has reported that ectopic expression of the four reprogramming factors for a short time in tail tip fibroblasts could be employed as a "shortcut" to mouse cardiogenesis. ${ }^{29}$ Recently, partial direct reprogramming of mature amniotic cells into ECs was also achieved. It was specifically reported that human amniotic cells could be effectively reprogrammed into vascular ECs (reprogrammed amniotic fluid-derived cells into vascular 


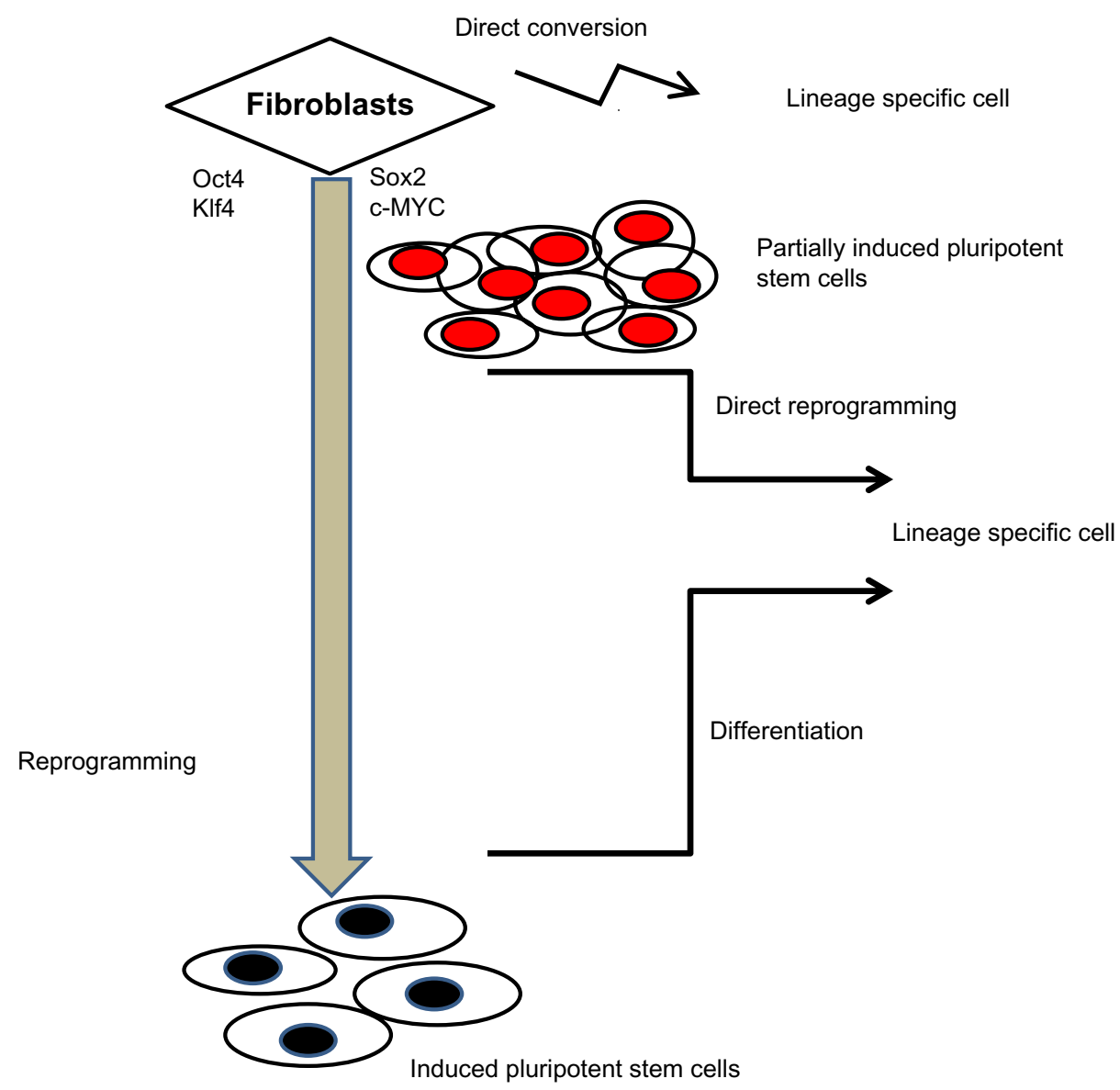

Figure I Schematic diagram showing reprogramming of fibroblasts to induced pluripotent stem cells followed by differentiation, direct reprogramming of fibroblasts with four factors generating Partial-iPS cells, and direct conversion.

Abbreviations: c-MYC, c-myelocytomatosis oncogene; KIf4, Kruppel-like factor 4; Oct4, octamer-binding protein; Sox2, sex determining region Y-box containing gene 2.

endothelial cells [rAC-VECs]), without passing through a pluripotent state. They specifically showed that using ETS transcription factors (ETV2, FLI1, and ERG1) could induce rAC-VECs, and transient inhibition of transforming growth factor- $\beta$ (TGF $\beta$ ) stabilized their vascular identity. Functionally, rAC-VECs were able to achieve in vitro tubulogenesis as well as form stable vasculatures in Matrigel plugs. ${ }^{64}$

\section{Clinical applications}

Despite ongoing clinical trials using regenerative therapy, the repair and regeneration of cells and tissues in clinical application still faces many hurdles. One of the major obstacles is the reduced availability of suitable cells needed for therapeutic purposes. A key example highlighting the need for readily-available sources of a large number of desired cells can be found in the field of cardiac disease (and atherosclerosis), which is a leading cause of mortality and morbidity worldwide ${ }^{65}$ Regenerative capacity is limited in the adult heart, ${ }^{66}$ which necessitates the urgent development of fast and robust new therapies. It is important to note that vascular endothelium is central to cardiovascular homeostasis, ${ }^{67}$ while chronic inflammation of the arterial wall, a common manifestation in atherosclerosis and an early event in disease processes, ${ }^{68}$ is initiated by structural alterations and EC dysfunction. ${ }^{69}$ In addition, alterations in $\mathrm{EC}$ function facilitate the infiltration of inflammatory cells and regulate vascular smooth muscle proliferation and platelet aggregation. ${ }^{70}$ Therefore, the generation of large numbers of ECs, usually limited in number in human patients, would be greatly beneficial in combating the disease in a clinical context.

\section{Limitations}

A number of technical hurdles need to be overcome before generating large quantities of the desired cell type. Some of the most common issues include the relatively low conversion efficiency from one type to another as well as the generation of a heterogeneous cell population, even after purification. Another factor that also needs consideration is the limited scalability of this cell generation system. Just like in transdifferentiation, the lack of a proliferative 
precursor stage compromises the cell-generating capacity of the system and, thus, the number of postmitotic cells is relatively low and even finite. ${ }^{71}$ In addition to the above, before introducing direct reprogramming in clinical trials, another limitation that needs to be considered is that the introduction of viruses into human subjects is generally undesirable; the mechanisms of viral action in humans remain unclear and such usage could introduce unpredictable factors that could lead to infections or even cancer. ${ }^{72}$ This substantiates the need to consider other means of delivering reprogramming factors, such as, via specially modified RNA or small molecules. ${ }^{73}$ Last but not of least importance, certain human cell types, such as fibroblasts, are usually more difficult to reprogram compared, for example, to mouse fibroblasts. ${ }^{74}$ Therefore, the following variables merit great importance: the starting cell number, the conversion capacity of the cells, and the efficiency of the executed reprogramming protocol.

\section{Alternative avenues}

Combinational strategies have been used to evaluate the use of specific miRNAs (miR-1, miR-133, miR-208, and miR499 ) for the in vitro induction of direct cell reprogramming of fibroblasts to cardiomyocyte-like cells. Indeed, transient expression of the miRNAs directed a cell fate switch, while the miRNA-transfected cardiac fibroblasts showed spontaneous calcium oscillations and transients in response to depolarization. ${ }^{54}$ When miRNAs were injected into the myocardium of ischemic mice in vivo, a direct conversion of fibroblasts to cardiomyocytes was observed. ${ }^{54}$ In addition, it was also reported that cardiomyocytes were generated by direct reprogramming of human fibroblasts using myocardin, miR-1, miR-133, and Hand2 plus GMT (GHMT) after several weeks in cell culture. However, the efficiency of conversion to functional cells (as shown by calcium transient measurements and spontaneous contraction) was comparatively lower to that of mouse fibroblasts, and extended cell culture maturation had to be employed. ${ }^{29}$ Until recently, the usual route for the evaluation of reprogramming has depended on nonfunctional measures such as flow cytometry or expression of green fluorescent protein (GFP). Using calcium activity, several known and novel combinations of transcription factors were compared in mouse embryonic fibroblasts. It was found that the most efficient combination for generating cardiomyocytelike cells with cardiomyocyte marker expression, consisted of Hand2, NK2 homeobox 5 (Nkx2.5), GATA4, Mef2c, and Tbx5 (HNGMT) and was $>50$-fold more efficient than GMT alone. ${ }^{75}$ Epigenetics are also of importance when it comes to reprogramming. Efficiency of reprogramming was shown to be improved by compounds that modulate epigenetic-related enzymes, which include histone deacetylases, histone methyltransferases, and DNA methyltransferases. ${ }^{76}$ However, it is difficult in this case to assess specificity in inducing key reprogramming factors due to the fact that further studies are needed to elucidate reprogramming mechanisms.

For example, it was found that BIX-01294, an inhibitor of the histone methyltransferase EHMT2, could enhance Oct4, Klf4-mediated reprogramming of neural progenitor cells into iPS cells, ending up with levels comparable to those of the Oct4/Sox2/K1f4/c-Myc-based reprogramming, ${ }^{77}$ albeit with reduced efficiency. Histone deacetylase inhibitors were also reported to improve reprogramming efficiency, ${ }^{78,79}$ while in another example, valproic acid, another histone deacetylase inhibitor, has been used in the reprogramming of human fibroblasts with Oct4 and Sox $2 .{ }^{80}$ Therefore, it can be assumed that, apart from assisting in the reprogramming toward a pluripotent stage, epigenetics may also enhance the direct reprogramming and direct conversion from one cell type to the next.

\section{Signaling pathways and small molecules}

Signal pathways, along with their modulators, have also proven helpful during cellular reprogramming. For example, the Wnt- $\beta$-catenin signaling pathway enhanced reprogramming by lessening the inhibition by $\mathrm{T}$ cell factor- 3 on pluripotency. ${ }^{81}$ In addition, a glycogen synthase kinase 3 inhibitor, CHIR99021, which can activate Wnt signaling, helped in reprogramming mouse embryonic fibroblasts (MEFs) to iPS cells through overexpression of Oct4 and Klf4. ${ }^{82}$ Similarly, it also helped with reprogramming human keratinocytes when in combination with an inhibitor of lysine specific demethylase $1{ }^{82}$ In addition, kenpaullone, an inhibitor of glycogen synthase kinase 3, was also shown to replace Klf4 when combined with the other three reprogramming factors Oct4, Sox2, and C-Myc during MEFs reprogramming. ${ }^{83}$ Furthermore, TGF $\beta$ signaling is important in inducing epithelial-mesenchymal transition during embryonic development. ${ }^{84}$ The reverse process of inhibiting TGF $\beta$ can thus be a central and decisive event toward pluripotency: it is expected that inhibitors of TGF $\beta$ signaling can facilitate this reversal and improve reprogramming. For example, TGF $\beta$ receptor inhibitors can indeed enhance reprogramming and even replace Sox 2 in MEFs. ${ }^{85,86}$ In a similar fashion, small molecules inhibiting TGF $\beta$ receptors, mitogen-activated protein kinase and Rho-associated protein kinase, were found to both improve and fast-track the reprogramming efficiency in 
human fibroblasts. ${ }^{87}$ In another example, forskolin and dorsomorphin were shown to assist neurogenin 2 in efficiently and directly converting human lung fibroblasts into neurons with high purity, bypassing the proliferative progenitor state. When transcription factor Sox11 was included, it helped in efficiently converting human skin fibroblasts derived from healthy and unhealthy patients to cholinergic neurons. ${ }^{88}$ It is also important to note that compounds that stimulate glycolytic metabolism have also been reported to improve reprogramming - for example, fructose 2,6-bisphosphate, which activates the phosphofructokinase 1 enzyme in a rate-limiting glycolytic step, or N-oxaloylglycine and quercetin, which activate hypoxia inducible factor- $1 .{ }^{89}$ Metabolic modulation of genes and pathways may, therefore, also be considered as an alternative means toward improving cellular reprogramming. Last but not least, a promising route that may increase the efficiency of direct reprogramming would be hypoxic conditions. Previous studies have shown that hypoxia has enhanced reprogramming by stimulating associated growth factor production. ${ }^{90}$

\section{Physical enhancement of direct reprogramming}

Cellular reprogramming may also be improved using custommade biomaterials. For example, instead of using Matrigelcoated tissue culture polystyrene, a rigid material that may nonspecifically absorb serum proteins, polyethylene glycol materials that are protein absorption-resistant can be modified and used in its place. It was, thus, shown that polyethylene glycol hydrogels improved both proliferation and reprogramming efficiency, almost doubling the cardiomyocyte-like cells that were sarcomeric $\alpha$-actinin-positive, compared to the original studies. ${ }^{91}$ In another study, the key, reprogramming gene Sox 2 was overexpressed in mouse fibroblasts cultured in a three-dimensional-spherical manner giving rise to neuron progenitor-like cells in vitro, which were then able to differentiate into specific neuron cell types such as neurons, astrocytes, and oligodendrocytes. In addition, after in vivo engraftment into adult rat brain, the three-dimensional cellular spheres differentiated into neural cells, showing that neural progenitor cells can be directly derived from fibroblasts using a physical approach devoid of exogenous transcription factors..$^{92}$

Even though functional retinal pigment epithelium (RPE) cells can be derived from either ESCs or iPS cells, direct reprogramming could greatly facilitate their generation. Using a human RPE-specific reporter (Best1::GFP) it was also found that cells positive for Best $1:$ GFP were able to form colonies and display characteristics of early-stage RPE cells. Furthermore, they acquired pigmentation after activation of retinoic acid and sonic hedgehog signal pathways..$^{93}$

\section{Summary and future directions}

Several of the latest advances in regenerative cell technology have alleviated some of the concerns of retroviral usage by introducing new methodologies to deliver reprogramming factors to cells. Such methods include episomal plasmids, ${ }^{94}$ excisable expression systems, ${ }^{95}$ messenger RNAs and miRNAs, ${ }^{96-99}$ or cell-penetrating recombinant proteins. ${ }^{100}$ Even though recent technical advances with iPS cell reprogramming have shown much promise, it is still considered slow and inefficient. The technology is still a long way from being a highly specific and directed event. Similarly, when it comes to direct cellular conversion with transdifferentiation, the resulting limited capacity for proliferation and cell diversity may markedly compromise any applications in regenerationbased therapies. Thus, it is important to consider additional avenues in cellular reprogramming, for example, through partial direct reprogramming assisted by the appropriate use of relevant master genes, specific small molecules, or even physical-based enhancements. Such alternative methods and advances could possibly address the safety issues associated with ESCs/iPS cells and the epigenetic changes occurring during cell reprogramming. ${ }^{101,102}$ This could be achieved by avoiding the viral vector-generated iPS cells, where reprogramming factors integrate into the host genome and may increase tumorigenicity, immunogenicity, genetic/ epigenetic abnormalities, ${ }^{24}$ or ESCs' tumorigenic potential ${ }^{103}$ in transplanted cells. By examining and altering the differentiated state, new avenues may widen prospects for the generation of novel research tools and therapeutic resources. The end products of such a reprogramming practice could be then directly implemented in regenerative and personalized medicine.

\section{Disclosure}

The authors report no conflicts of interest in this work.

\section{References}

1. Ladewig J, Koch P, Brüstle O. Leveling Waddington: the emergence of direct programming and the loss of cell fate hierarchies. Nat Rev Mol Cell Biol. 2013;14(4):225-236.

2. Patel M, Yang S. Advances in reprogramming somatic cells to induced pluripotent stem cells. Stem Cell Rev. 2010;6(3):367-380.

3. Boiani M, Schöler HR. Regulatory networks in embryo-derived pluripotent stem cells. Nat Rev Mol Cell Biol. 2005;6(11):872-884.

4. Gepstein L. Derivation and potential applications of human embryonic stem cells. Circ Res. 2002;91(10):866-876.

5. Nakagami H, Nakagawa N, Takeya Y, et al. Model of vasculogenesis from embryonic stem cells for vascular research and regenerative medicine. Hypertension. 2006;48(1):112-119. 
6. Brunt KR, Weisel RD, Li RK. Stem cells and regenerative medicine future perspectives. Can J Physiol Pharmacol. 2012;90(3):327-335.

7. Atala A. Regenerative medicine strategies. J Pediatr Surg. 2012; 47(1):17-28.

8. Keller G. Embryonic stem cell differentiation: emergence of a new era in biology and medicine. Genes Dev. 2005;19(10):1129-1155.

9. Pillai RG. Stem cells for ocular tissue engineering and regeneration. Curr Top Med Chem. 2011;11(13):1606-1620.

10. Pappa KI, Anagnou NP. Novel sources of fetal stem cells: where do they fit on the developmental continuum? Regen Med. 2009;4(3): 423-433.

11. Yen BL, Yen ML, Hsu PJ, et al. Multipotent human mesenchymal stromal cells mediate expansion of myeloid-derived suppressor cells via hepatocyte growth factor/c-Met and STAT3. Stem Cell Reports. 2013;1(2):139-151.

12. Lengner CJ. iPS cell technology in regenerative medicine. Ann NY Acad Sci. 2010;1192:38-44.

13. Takahashi K, Yamanaka S. Induction of pluripotent stem cells from mouse embryonic and adult fibroblast cultures by defined factors. Cell. 2006;126(4):663-676.

14. Chin MH, Mason MJ, Xie W, et al. Induced pluripotent stem cells and embryonic stem cells are distinguished by gene expression signatures. Cell Stem Cell. 2009;5(1):111-123.

15. Saric T, Hescheler J. Stem cells and nuclear reprogramming. Minim Invasive Ther Allied Technol. 2008;17(2):64-78.

16. Bilic J, Izpisua Belmonte JC. Concise review: Induced pluripotent stem cells versus embryonic stem cells: close enough or yet too far apart? Stem Cells. 2012;30(1):33-41.

17. Yu J, Thomson JA. Pluripotent stem cell lines. Genes Dev. 2008;22(15): 1987-1997.

18. Rufaihah AJ, Huang NF, Kim J, et al. Human induced pluripotent stem cell-derived endothelial cells exhibit functional heterogeneity. $\mathrm{Am} \mathrm{J}$ Transl Res. 2013;5(1):21-35.

19. Hu BY, Weick JP, Yu J, et al. Neural differentiation of human induced pluripotent stem cells follows developmental principles but with variable potency. Proc Natl Acad Sci U S A. 2010;107(9):4335-4340.

20. Zhang J, Wilson GF, Soerens AG, et al. Functional cardiomyocytes derived from human induced pluripotent stem cells. Circ Res. 2009;104(4):e30-e41.

21. Azhdari M, Baghaban-Eslaminejad M, Baharvand H, Aghdami N. Therapeutic potential of human-induced pluripotent stem cell-derived endothelial cells in a bleomycin-induced scleroderma mouse model. Stem Cell Res. 2013;10(3):288-300.

22. Juopperi TA, Song H, Ming GL. Modeling neurological diseases using patient-derived induced pluripotent stem cells. Future Neurol. 2011;6(3):363-373.

23. Collado M, Blasco MA, Serrano M. Cellular senescence in cancer and aging. Cell. 2007;130(2):223-233.

24. Okano H, Nakamura M, Yoshida K, et al. Steps toward safe cell therapy using induced pluripotent stem cells. Circ Res. 2013;112(3): 523-533.

25. Solter D. Mammalian cloning: advances and limitations. Nat Rev Genet. 2000;1(3):199-207.

26. Vierbuchen T, Ostermeier A, Pang ZP, Kokubu Y, Südhof TC, Wernig M. Direct conversion of fibroblasts to functional neurons by defined factors. Nature. 2010;463(7284):1035-1041.

27. Ieda M, Fu JD, Delgado-Olguin $\mathrm{P}$, et al. Direct reprogramming of fibroblasts into functional cardiomyocytes by defined factors. Cell. 2010;142(3):375-386.

28. Sekiya S, Suzuki A. Direct conversion of mouse fibroblasts to hepatocytelike cells by defined factors. Nature. 2011;475(7356):390-393.

29. Nam YJ, Song K, Luo X, et al. Reprogramming of human fibroblasts toward a cardiac fate. Proc Natl Acad Sci U S A. 2013;110(14): 5588-5593.

30. Qian L, Huang Y, Spencer CI, et al. In vivo reprogramming of murine cardiac fibroblasts into induced cardiomyocytes. Nature. 2012;485(7400):593-598.
31. Gehring WJ. The master control gene for morphogenesis and evolution of the eye. Genes Cells. 1996;1(1):11-15.

32. Schneuwly S, Klemenz R, Gehring WJ. Redesigning the body plan of Drosophila by ectopic expression of the homoeotic gene Antennapedia. Nature. 1987;325(6107):816-818.

33. Yamanaka S, Blau HM. Nuclear reprogramming to a pluripotent state by three approaches. Nature. 2010;465(7299):704-712.

34. Davis RL, Weintraub H, Lassar AB. Expression of a single transfected cDNA converts fibroblasts to myoblasts. Cell. 1987;51(6):987-1000.

35. Gurdon JB. Adult frogs derived from the nuclei of single somatic cells. Dev Biol. 1962;4:256-273.

36. Blau HM, Pavlath GK, Hardeman EC, et al. Plasticity of the differentiated state. Science. 1985;230(4727):758-766.

37. Briggs R, King TJ. Transplantation of living nuclei from blastula cells into enucleated frogs' eggs. Proc Natl Acad Sci U SA. 1952;38(5):455-463.

38. Choi J, Costa ML, Mermelstein CS, Chagas C, Holtzer S, Holtzer H. MyoD converts primary dermal fibroblasts, chondroblasts, smooth muscle, and retinal pigmented epithelial cells into striated mononucleated myoblasts and multinucleated myotubes. Proc Natl Acad Sci U S A. 1990;87(20):7988-7992.

39. Schäfer BW, Blakely BT, Darlington GJ, Blau HM. Effect of cell history on response to helix-loop-helix family of myogenic regulators. Nature. 1990;344(6265):454-458.

40. Kulessa H, Frampton J, Graf T. GATA-1 reprograms avian myelomonocytic cell lines into eosinophils, thromboblasts, and erythroblasts. Genes Dev. 1995;9(10):1250-1262.

41. Xie H, Ye M, Feng R, Graf T. Stepwise reprogramming of B cells into macrophages. Cell. 2004;117(5):663-676.

42. Nutt SL, Heavey B, Rolink AG, Busslinger M. Commitment to the B-lymphoid lineage depends on the transcription factor Pax5. Nature. 1999;401(6753):556-562.

43. Cobaleda C, Jochum W, Busslinger M. Conversion of mature B cells into $\mathrm{T}$ cells by dedifferentiation to uncommitted progenitors. Nature. 2007;449(7161):473-477.

44. Zhou Q, Brown J, Kanarek A, Rajagopal J, Melton DA. In vivo reprogramming of adult pancreatic exocrine cells to beta-cells. Nature. 2008;455(7213):627-632.

45. Song K, Nam YJ, Luo X, et al. Heart repair by reprogramming nonmyocytes with cardiac transcription factors. Nature. 2012;485(7400): 599-604.

46. Protze S, Khattak S, Poulet C, Lindemann D, Tanaka EM, Ravens U. A new approach to transcription factor screening for reprogramming of fibroblasts to cardiomyocyte-like cells. J Mol Cell Cardiol. 2012;53(3): 323-332.

47. Addis RC, Epstein JA. Induced regeneration - the progress and promise of direct reprogramming for heart repair. Nat Med. 2013;19(7): $829-836$.

48. Inagawa $\mathrm{K}$, Miyamoto $\mathrm{K}$, Yamakawa $\mathrm{H}$, et al. Induction of cardiomyocyte-like cells in infarct hearts by gene transfer of Gata4, Mef2c, and Tbx5. Circ Res. 2012;111(9):1147-1156.

49. Mathison M, Gersch RP, Nasser A, et al. In vivo cardiac cellular reprogramming efficacy is enhanced by angiogenic preconditioning of the infarcted myocardium with vascular endothelial growth factor. $J \mathrm{Am}$ Heart Assoc. 2012;1(6):e005652.

50. Mummery C, Ward-van Oostwaard D, Doevendans P, et al. Differentiation of human embryonic stem cells to cardiomyocytes: role of coculture with visceral endoderm-like cells. Circulation. 2003; 107(21):2733-2740

51. Narazaki G, Uosaki H, Teranishi M, et al. Directed and systematic differentiation of cardiovascular cells from mouse induced pluripotent stem cells. Circulation. 2008;118(5):498-506.

52. Chen JX, Krane M, Deutsch MA, et al. Inefficient reprogramming of fibroblasts into cardiomyocytes using Gata4, Mef2c, and Tbx5. Circ Res. 2012;111(1):50-55.

53. Yi BA, Mummery CL, Chien KR. Direct cardiomyocyte reprogramming: a new direction for cardiovascular regenerative medicine. Cold Spring Harb Perspect Med. 2013;3(9):a014050. 
54. Jayawardena TM, Egemnazarov B, Finch EA, et al. MicroRNAmediated in vitro and in vivo direct reprogramming of cardiac fibroblasts to cardiomyocytes. Circ Res. 2012;110(11):1465-1473.

55. Margariti A, Winkler B, Karamariti E, et al. Direct reprogramming of fibroblasts into endothelial cells capable of angiogenesis and reendothelialization in tissue-engineered vessels. Proc Natl Acad Sci U SA. 2012;109(34):13793-13798.

56. Graf T, Enver T. Forcing cells to change lineages. Nature. 2009; 462(7273):587-594.

57. Ho L, Crabtree GR. Chromatin remodelling during development. Nature. 2010;463(7280):474-484.

58. Takahashi K, Okita K, Nakagawa M, Yamanaka S. Induction of pluripotent stem cells from fibroblast cultures. Nat Protoc. 2007;2(12): 3081-3089.

59. Cherry AB, Daley GQ. Reprogramming cellular identity for regenerative medicine. Cell. 2012;148(6):1110-1122.

60. Sheridan WS, Duffy GP, Murphy BP. Mechanical characterization of a customized decellularized scaffold for vascular tissue engineering. J Mech Behav Biomed Mater. 2012;8:58-70.

61. Novosel EC, Kleinhans C, Kluger PJ. Vascularization is the key challenge in tissue engineering. Adv Drug Deliv Rev. 2011;63(4-5): 300-311.

62. Li J, Huang NF, Zou J, et al. Conversion of human fibroblasts to functional endothelial cells by defined factors. Arterioscler Thromb Vasc Biol. 2013;33(6):1366-1375.

63. Karamariti E, Margariti A, Winkler B, et al. Smooth muscle cells differentiated from reprogrammed embryonic lung fibroblasts through DKK3 signaling are potent for tissue engineering of vascular grafts. Circ Res. 2013;112(11):1433-1443.

64. Ginsberg M, James D, Ding BS, et al. Efficient direct reprogramming of mature amniotic cells into endothelial cells by ETS factors and TGF $\beta$ suppression. Cell. 2012;151(3):559-575.

65. Ignarro LJ, Balestrieri ML, Napoli C. Nutrition, physical activity, and cardiovascular disease: an update. Cardiovasc Res. 2007;73(2): 326-340.

66. Rasmussen TL, Raveendran G, Zhang J, Garry DJ. Getting to the heart of myocardial stem cells and cell therapy. Circulation. 2011;123(16):1771-1779.

67. Cines DB, Pollak ES, Buck CA, et al. Endothelial cells in physiology and in the pathophysiology of vascular disorders. Blood. 1998;91(10): 3527-3561.

68. Davignon J, Ganz P. Role of endothelial dysfunction in atherosclerosis. Circulation. 2004;109(23 Suppl 1):III27-III32.

69. Weber C, Noels H. Atherosclerosis: current pathogenesis and therapeutic options. Nat Med. 2011;17(11):1410-1422.

70. Wong WT, Huang NF, Botham CM, Sayed N, Cooke JP. Endothelial cells derived from nuclear reprogramming. Circ Res. 2012;111(10): 1363-1375.

71. Pawlowski M, Kotter M. Generation of neural cells by direct cellular reprogramming. J Transplant Stem Cell Biol. 2013;1(1):7.

72. Butel JS. Viral carcinogenesis: revelation of molecular mechanisms and etiology of human disease. Carcinogenesis. 2000;21(3):405-426.

73. Srivastava D, Ieda M. Critical factors for cardiac reprogramming. Circ Res. 2012;111(1):5-8.

74. Yan X, Qin H, Qu C, Tuan RS, Shi S, Huang GT. iPS cells reprogrammed from human mesenchymal-like stem/progenitor cells of dental tissue origin. Stem Cells Dev. 2010;19(4):469-480.

75. Addis RC, Ifkovits JL, Pinto F, et al. Optimization of direct fibroblast reprogramming to cardiomyocytes using calcium activity as a functional measure of success. J Mol Cell Cardiol. 2013;60:97-106.

76. Zhang Y, Li W, Laurent T, Ding S. Small molecules, big roles - the chemical manipulation of stem cell fate and somatic cell reprogramming. $J$ Cell Sci. 2012;125(Pt 23):5609-5620.

77. Shi Y, Desponts C, Do JT, Hahm HS, Schöler HR, Ding S. Induction of pluripotent stem cells from mouse embryonic fibroblasts by Oct 4 and Klf4 with small-molecule compounds. Cell Stem Cell. 2008;3(5): $568-574$.
78. Sun G, Fu C, Shen C, Shi Y. Histone deacetylases in neural stem cells and induced pluripotent stem cells. J Biomed Biotechnol. 2011;2011:835968.

79. Mikkelsen TS, Hanna J, Zhang X, et al. Dissecting direct reprogramming through integrative genomic analysis. Nature. 2008;454(7200): 49-55.

80. Huangfu D, Osafune K, Maehr R, et al. Induction of pluripotent stem cells from primary human fibroblasts with only Oct4 and Sox2. Nat Biotechnol. 2008;26(11):1269-1275.

81. Niwa H. Wnt: what's needed to maintain pluripotency? Nat Cell Biol. 2011;13(9):1024-1026.

82. Li W, Zhou H, Abujarour R, et al. Generation of human-induced pluripotent stem cells in the absence of exogenous Sox2. Stem Cells. 2009;27(12):2992-3000.

83. Lyssiotis CA, Foreman RK, Staerk J, et al. Reprogramming of murine fibroblasts to induced pluripotent stem cells with chemical complementation of Klf4. Proc Natl Acad Sci U S A. 2009;106(22):8912-8917.

84. Xie L, Law BK, Chytil AM, Brown KA, Aakre ME, Moses HL. Activation of the Erk pathway is required for TGF-beta1-induced EMT in vitro. Neoplasia. 2004;6(5):603-610.

85. Ichida JK, Blanchard J, Lam K, et al. A small-molecule inhibitor of tgf-Beta signaling replaces sox 2 in reprogramming by inducing nanog. Cell Stem Cell. 2009;5(5):491-503.

86. Maherali N, Hochedlinger K. Tgfbeta signal inhibition cooperates in the induction of iPSCs and replaces Sox 2 and cMyc. Curr Biol. 2009;19(20):1718-1723.

87. Lin T, Ambasudhan R, Yuan X, et al. A chemical platform for improved induction of human iPSCs. Nat Methods. 2009;6(11):805-808.

88. Liu ML, Zang T, Zou Y, et al. Small molecules enable neurogenin 2 to efficiently convert human fibroblasts into cholinergic neurons. Nat Commun. 2013;4:2183.

89. Zhu S, Li W, Zhou H, et al. Reprogramming of human primary somatic cells by OCT4 and chemical compounds. Cell Stem Cell. 2010;7(6):651-655.

90. Yoshida Y, Takahashi K, Okita K, Ichisaka T, Yamanaka S. Hypoxia enhances the generation of induced pluripotent stem cells. Cell Stem Cell. 2009;5(3):237-241.

91. Smith AW, Hoyne JD, Nguyen PK, et al. Direct reprogramming of mouse fibroblasts to cardiomyocyte-like cells using Yamanaka factors on engineered poly(ethylene glycol) (PEG) hydrogels. Biomaterials. 2013;34(28):6559-6571.

92. Su G, Zhao Y, Wei J, et al. Direct conversion of fibroblasts into neural progenitor-like cells by forced growth into 3D spheres on low attachment surfaces. Biomaterials. 2013;34(24):5897-5906.

93. Zhang K, Liu GH, Yi F, et al. Direct conversion of human fibroblasts into retinal pigment epithelium-like cells by defined factors. Protein Cell. Epub June 20, 2013.

94. Yu J, Vodyanik MA, Smuga-Otto K, et al. Induced pluripotent stem cell lines derived from human somatic cells. Science. 2007;318(5858): 1917-1920.

95. Soldner F, Hockemeyer D, Beard C, et al. Parkinson's disease patientderived induced pluripotent stem cells free of viral reprogramming factors. Cell. 2009;136(5):964-977.

96. Warren L, Manos PD, Ahfeldt T, et al. Highly efficient reprogramming to pluripotency and directed differentiation of human cells with synthetic modified mRNA. Cell Stem Cell. 2010;7(5):618-630.

97. Yakubov E, Rechavi G, Rozenblatt S, Givol D. Reprogramming of human fibroblasts to pluripotent stem cells using mRNA of four transcription factors. Biochem Biophys Res Commun. 2010;394(1):189-193

98. Anokye-Danso F, Trivedi CM, Juhr D, et al. Highly efficient miRNAmediated reprogramming of mouse and human somatic cells to pluripotency. Cell Stem Cell. 2011;8(4):376-388.

99. Miyoshi N, Ishii H, Nagano H, et al. Reprogramming of mouse and human cells to pluripotency using mature microRNAs. Cell Stem Cell. 2011;8(6):633-638.

100. Zhou H, Wu S, Joo JY, et al. Generation of induced pluripotent stem cells using recombinant proteins. Cell Stem Cell. 2009;4(5):381-384. 
101. Kim K, Doi A, Wen B, et al. Epigenetic memory in induced pluripotent stem cells. Nature. 2010;467(7313):285-290.

102. Ohi Y, Qin H, Hong C, et al. Incomplete DNA methylation underlies a transcriptional memory of somatic cells in human iPS cells. Nat Cell Biol. 2011;13(5):541-549.
103. Blum B, Benvenisty N. The tumorigenicity of human embryonic stem cells. Adv Cancer Res. 2008;100:133-158.

\section{Publish your work in this journal}

Stem Cells and Cloning: Advances and Applications is an international, peer-reviewed, open access journal. Areas of interest in stem cell research include: Embryonic stem cells; Adult stem cells; Blastocysts; Cordblood stem cells; Stem cell transformation and culture; Therapeutic cloning; Umbilical cord blood and bone marrow cells; Laboratory, animal and human therapeutic studies; Philosophical and ethical issues related to stem cell research. This journal is indexed on CAS. The manuscript management system is completely online and includes a quick and fair peer-review system. Visit http://www.dovepress.com/ testimonials.php to read real quotes from published authors.

\footnotetext{
Submit your manuscript here: http://www.dovepress.com/stem-cells-and-cloning-advances-and-applications-journal
} 